\title{
Improvements in individual dose measurement techniques following nuclear emergencies
}

\author{
M.A. López ${ }^{1, *}$, V. Berkovskyy ${ }^{2}$, G. Ratia ${ }^{2}$, C. Challeton-de-Vathaire ${ }^{3}$, E. Davesne ${ }^{3}$, J. Eakins ${ }^{4}$, \\ D. Franck ${ }^{3}$, A. Giussani ${ }^{5}$, D. Gregoratto ${ }^{4}$, C. Hernandez ${ }^{1}$, U. Kulka ${ }^{5}$, A. Mafodda ${ }^{6}$, J.W. Marsh ${ }^{4}$, \\ J.F. Navarro ${ }^{1}$, U. Oestreicher ${ }^{5}$, B. Pérez ${ }^{1}$, I. Sierra ${ }^{1}$ and C. Woda ${ }^{6}$ \\ ${ }^{1}$ CIEMAT - Centro de Investigaciones Energéticas, Medioambientales y Tecnológicas, Madrid, Spain. \\ 2 RPI, Kyiv, Ukraine. \\ 3 IRSN - Institut for Radioprotection and Nuclear Safety, Fontenay-aux-Roses, France. \\ ${ }^{4}$ PHE - Public Health England, Centre for Radiation, Chilton, Didcot, UK. \\ ${ }^{5}$ BfS - Federal Office for Radiation Protection, Oberschleißheim, Germany. \\ ${ }^{6}$ HMGU - Helmholtz Zentrum München - German Research Center for Environmental Health, Neuherberg, Germany.
}

\begin{abstract}
The aim of CONFIDENCE WP2 is to improve the situation awareness in the early phase of a nuclear accident by trying to reduce the uncertainty in individual dose assessment when dealing with external and internal exposures. Main WP2 research actions to improve external dosimetry in this emergency frame were the development of destruction-free protocols using electronic components in smartphones for external dose measurements, Monte Carlo (MCNP) calculations for organ dose assessment with associated uncertainties and the organization of a workshop for integration of biodosimetry into emergency response. On the other hand, one of the main concerns after the release of radioactive material in case of a nuclear reactor accident is the intake of radioiodines in workers and population. A smartphone/ tablet application for direct calculation of thyroid doses from monitoring data of the content of ${ }^{131} \mathrm{I}\left(\right.$ and $\left.{ }^{132} \mathrm{I}\right)$ in the thyroid was developed during the project (Prototype of processing unit for thyroid dose monitor [IDOSE]). Up to date age-dependent ICRP dose per content values are used by this tool, allowing a rapid screening of exposed persons. A sensitivity analysis on thyroid doses was carried out, considering 16 exposure scenarios leading to 16 different dose estimates, using ICRP56/119 (ICRP60) vs. ICRP130/137 iodine models for adults, varying the time of intake, the time pattern (acute $v s$. chronic) and considering not well defined relative abundance of short-lived radioiodines and ${ }^{132} \mathrm{Te}$. Real cases of Europeans contaminated in Japan shortly after the Fukushima NPP accident (low doses), and artificial cases of high doses generated to see the impact of the different parameters in this study, were used for applying this multiintake scenario approach. Main conclusions of the study are presented here.
\end{abstract}

Keywords: individual dosimetry / thyroid doses / retrospective dosimetry / biological dosimetry

\section{Introduction}

The aim of CONFIDENCE WP2 is to improve the situation awareness in the early phase of a nuclear accident by trying to reduce the uncertainty in individual dose assessment when dealing with internal and external exposures. Thyroid monitoring to quantify doses due to radioiodine intakes and retrospective dosimetry techniques using personal items and blood samples of exposed population are the dosimetry methods considered here for accidental exposures to gamma radiation.

\footnotetext{
*Corresponding author: ma.lopez@ciemat.es
}

\section{External dosimetry using personal items}

While methods for individual monitoring and assessing individual doses from internal contamination following a nuclear emergency are relatively advanced and matured, doses from external exposure are often only calculated from environmental data and methods of individual dose measurement are rarely applied in this case or are still under development. An established approach is biological dosimetry on peripheral blood samples, but in the past decade a number of items have also been identified that could be measured using physical retrospective dosimetry techniques. Therefore, a research on dosimetric properties of some personal items and 
their possible usage as fortuitous dosimeters during an emergency scenario has been conducted. In fact, electronic components of mobile phones (Surface Mount Resistors), chip and memory cards, are identified as valuable and challenging resource of information for individuals externally exposed to gamma radiation following a nuclear accident. Investigations on new retrospective dosimetry approaches on known materials (e.g. resistors) and on new materials that might enhance acceptability of the methods by the public (e.g. destructive protocols on less valuable materials) were carried out. Moreover, since in a nuclear emergency doses can be dominated by internal (inhalation and ingestion) doses and doses due to external exposure can be expected to be even lower, more emphasis was put on optimizing the techniques performance in the low dose range (20-200 mGy). On one hand, this challenge in terms of sensitivity was overcome by using the thermoluminescence (TL) technique on Surface Mount Resistors extracted from mobile phones in a new spectral detection window. The increase in sensitivity made it possible to develop a new protocol based on a single detector (single resistor of $1 \mathrm{~mm} \times 0.5 \mathrm{~mm}$ ), potentially enabling a sampling procedure where the component is replaced after measurement, thus leaving the phone functional and intact. In doing so, the issue of general acceptability of the method by the public is addressed. The TL technique was found to perform reliably down to $20 \mathrm{mGy}$, and a linear dose response was observed in the relevant dose range. However, the whole process of disassembling the phone - sampling - replacing the component - reassembling the phone can be time-consuming and not suitable for triage dosimetry, where it is required to process a large amount of samples in the shortest amount of time possible. Therefore, this approach seems primarily useful within the concept of targeting the critically exposed subgroups among the population affected by passage of a radioactive plume, after being selected for individualized dose calculations (e.g. in emergency care centres). In order to relate the absorbed doses into the resistors to the doses that are simultaneously received by the individuals "wearing" the phone, conversion factors sets were derived with the generalpurpose Monte Carlo radiation transport code MCNP6.2 (Werner, 2017).

Besides resistors, for which the dosimetry methods for reconstructing individual doses have been optimized in the low dose-range, chip cards and memory cards have shown their general use as a fortuitous dosimeter but need to be further investigated for application in the same low range. In literature, the minimum detectable dose for OSL (optically stimulated luminescence) of chip cards with a UV-cured transparent encapsulation is assessed as $2-7 \mathrm{mGy}$, immediately after irradiation (Cauwels et al., 2010; Woda and Spöttl, 2009). These values increase for dose measurements performed 10 days after irradiation, due to signal fading. Dose recovery tests carried out under idealized conditions, performed immediately and up to six days after irradiation, resulted to be successful on the reconstruction of the given doses within $10-15 \%$. For memory cards, the dose response of the OSL signal was shown to be linear up to at least $10 \mathrm{~Gy}$ and the detection limit was estimated at 20-40 mGy. After 10 days, also for such materials, fading becomes pronounced and leads to a signal loss of $\sim 80 \%$ and to an increase of the detection limit up to $100 \mathrm{mGy}$. Overall, reconstructing doses with memory cards was successful for doses of and above $2 \mathrm{~Gy}$. To conclude, external dosimetry using resistors from mobile phones in a potentially non-destructive approach could be possible with an uncertainty of 30-50\%, whereas reconstructing doses with less valuable personal items, such as memory cards, need to be further investigated in the low dose range.

\section{Integration of biodosimetry into emergency response}

A one-day workshop about integration of existing biodosimetry infrastructures into emergency response and Education\&Training programs was held on 30 September 2018 in Rovinj, Croatia, prior to the European Radiation Protection Week (ERPW2018 ${ }^{1}$ ). Participants were from organisations contributing to biological and physical retrospective dosimetry, especially in the RENEB (Running the European Network of Biological and retrospective Physical dosimetry $^{2}$ ) association (Kulka et al., 2017; Wojcik et al., 2017), among them representatives from BfS/Germany, IRSN/ France, PHE/United Kingdom, NRPA (now DSA)/Norway, $\mathrm{HGM}$ and UAB/Spain and SU/Sweden. The main objective of the workshop was how to better inform stakeholders and decision makers of existing concepts for biological and EPR/ OSL dosimetry. In particular, existing integration activities of biological and retrospective physical dosimetry in various European countries (e.g. Finland, France, Germany, Norway, Portugal, Spain, Sweden, United Kingdom, Austria, Switzerland) have been compiled and revised. While in some countries biological dosimetry is already integrated in emergency preparedness (e.g. in Germany), other countries do not have such options (e.g. Austria, Switzerland). In order to enable a uniform approach in Europe, it was decided to review the corresponding hierarchical structures and to establish joint cross boarder approaches, e.g. by following WHO recommendations. Various groups of people and organizations were identified during the workshop, which can contribute significantly to emergency preparedness as stakeholders on national and international level. At the national level, these are primarily doctors, especially involved in radiological emergencies and first aid facilities, as well as fire brigades and the Technical Relief Services (e.g. THW in Germany). At the international level, the IAEA and WHO are important contact points, which have already integrated biological dosimetry and physical retrospective dosimetry into their emergency programs (WHO BioDoseNet ${ }^{3}$, IAEA RANET $^{4}$ ), but also other regional and global networks contributing to individualized retrospective dosimerty (Kulka et al., 2019). It was decided to prepare and distribute information material such as flyers and short guides about individual retrospective dosimetry. This can be done by national authorities together with the RENEB association. The organization of regular inter-laboratory exercises for quality assurance is considered as core competence of the network to test the capabilities and reliability of the laboratories

\footnotetext{
${ }^{1}$ https://erpw2018.com/

${ }^{2}$ http://www.reneb.net/

${ }^{3} \mathrm{https}$ ://www.who.int/ionizing_radiation/a_e/biodosenet/en/

${ }^{4}$ https://www.iaea.org/services/networks/ranet
} 
(Oestreicher et al., 2017); the management of blood collection and the regulation of the sample shipment throughout Europe were topics of discussion. During the exercises various parameters may be tested, including transportation, processing and dose estimation of individualized samples after various irradiation scenarios. Here, the procedures within the RENEB network have been significantly harmonized, e.g. the calculation of the dose estimation, which was identified as a critical matter. The capabilities of the laboratories if necessary may be improved through training courses. To further harmonize dose estimation among laboratories, an online tool for dose calculation is under development and offered for free testing ("shinyapps") 5 . For general data collection, it was decided to prepare the STORE database ${ }^{6}$ for training and emergencies. This database has to be further developed to be used as a secure and reliable forum for training and for data collection in case of an emergency. Current concepts of biological dosimetry and retrospective physical dosimetry have been checked and options to optimize existing biodosimetry infrastructures have been discussed and identified. It was decided to continuously revise existing guidance documents, such as the MULTIBIODOSE guidance ${ }^{7}$ and national documents, to integrate activities of the RENEB network into national and international emergency response and E\&T programs.

\section{Internal exposures: thyroid doses and sensitivity analysis}

At the early phase of a nuclear emergency, one of the main concerns when dealing with internal exposures is to identify who are the most exposed individuals, to proceed with individual monitoring and to quantify their internal contamination level. This information may come from reliable environmental monitoring data using an appropriate tool developed in this project or from individual monitoring of gamma emitters in the body. The intake scenario to be considered here is acute inhalation or ingestion of ${ }^{131} \mathrm{I},{ }^{132} \mathrm{I}$, ${ }^{133} \mathrm{I},{ }^{135} \mathrm{I}$ and ${ }^{132} \mathrm{Te}$ after a nuclear reactor accident. A smart phone/tablet application was developed by the RPI (Ukraine) for direct thyroid dose evaluations using in vivo monitoring data of radioiodine in the thyroid (CONCERT Deliverable 9.9-Prototype of processing unit for thyroid dose monitor [IDOSE]). Dose assessments are based on "30-day thyroid absorbed dose per thyroid content" functions generated using the up to date ICRP biokinetic and dosimetric models for individuals of various ages, including embryo and foetus (see Fig. 1). The new ICRP/OIR model of radioiodine published in ICRP Publication 137 (ICRP, 2017), based on the methodologies from ICRP Publications 103 and 130 (ICRP, 2017, 2015, 2007), is used here for exposed adult people. Required input data are the measurement(s) results of ${ }^{131} \mathrm{I}$ and ${ }^{132}$ I activity content in thyroid, date and time of the nuclear accident, age/date of birth of the person or the gestational age of the foetus and the results of atmospheric/environmental

\footnotetext{
5 https://aldomann.shinyapps.io/biodose-tools-beta/_w_c43654c1/ w_70726150/_w_576296bf/

${ }^{\overline{6}}$ https://www.storedb.org/store_v3/

${ }^{7}$ http://www.reneb.net/wp-content/uploads/2017/09/multibiodoseguidance-small.pdf
}

transport modelling (ATM) with time-course (shape) of the inhalation/ingestion activity intake $(\mathrm{Bq})$ of ${ }^{131} \mathrm{I}$ and ${ }^{132} \mathrm{Te}$, associated with routes and places visited by the exposed individual. Output data are the 30-day committed absorbed dose to the thyroid of the exposed person (at term for the foetus) in Gy, the committed absorbed dose to the thyroid at the age of $70 \mathrm{y}$ in Gy and the committed effective dose in Sv. This IDOSE tool allows a rapid screening of a large number of exposed individuals.

A sensitivity analysis was carried out considering all the uncertainty sources related to measurements of radioiodine content in the thyroid gland and in dose assessment. Taking into account that Dose Per Content (DPC) function is insensitive to physico-chemical properties of the incorporated material (e.g. absorption type, particle size) and to route of intake (inhalation, ingestion), the important sources of uncertainty here are the following:

- uncertainty on the measurement of ${ }^{131} \mathrm{I}$ in the thyroid. Using the Scattering Factor (SF) approach for type A and type B uncertainties according to IDEAS Guidelines V2 (Castellani et al., 2013), SF is in the range of 1.15-1.20 and the quantities to be used for dose assessment are: nominal value $\mathrm{M}(\mathrm{Bq})$ of ${ }^{131} \mathrm{I}$ in the thyroid, upper limit $\left(\mathrm{M} \times \mathrm{SF}^{2}\right) \mathrm{Bq}$ and lower limit $\left(\mathrm{M} / \mathrm{SF}^{2}\right) \mathrm{Bq}$;

- unknown time of the intake, unknown intake pattern (acute or chronic), not well-defined relative abundance of short-lived radioiodines and different retention models and dose-percontent coefficients (based on ICRP Publication 119 [ICRP, 2012] vs., ICRP Publication 137 [ICRP, 2017], for adults).

Thyroid monitoring data from Europeans contaminated in Japan shortly after the releases of the Fukushima Daiichi NPP (low doses, Lopez et al., 2016; Champion et al., 2013) were used to complement the sensitivity study, considering 16 exposure scenarios leading to 16 different dose estimates of low level doses. This approach was used in case of a Czech volunteer in Ibaraki and Sendai locations in 20-28 March 2011, travelling to Tokyo on 28 March and finally to Prague where the in vivo monitoring took place on 6 April 2011 with a result of $52.0 \pm 9.0 \mathrm{~Bq}(2 \sigma)$ of ${ }^{131} \mathrm{I}$ in the thyroid. Higher dose (Hthy= $1.26 \mathrm{E}-4 \mathrm{~Sv}$ ) would correspond to an acute intake on $20 \mathrm{March}$ in Mito (Ibaraki), lower dose (Hthy $=3.72 \mathrm{E}-4 \mathrm{~Sv}$ ) would be associated to a $24 \mathrm{~h}$ chronic intake on 31 March in Tokyo.

Dose per thyroid content $z(t)$ values for adults, in the 16 scenarios defined before, were compared using ICRP Publication 119 (ICRP, 2012) dosimetric data $v s$. those from ICRP/OIR Publications, resulting in lower values for the latter (so lower thyroid doses when using ICRP Publication 137 [ICRP, 2017]).

Artificial cases were also generated to see the impact of the different parameters in this study for high level radioiodine intakes, applying this multi-intake scenario approach. Considering the case of a young female ( 20 years old) and multiple monitoring data of ${ }^{131} \mathrm{I}$ in the thyroid $\left(\mathrm{M}_{1}=1.6 \mathrm{E} 4 \pm 3.2 \mathrm{E}+2\right.$ $\left.\mathrm{Bq}, \mathrm{M}_{2}=1.0 \mathrm{E}+4 \pm 2 \mathrm{E}+2 \mathrm{~Bq}, \mathrm{M}_{3}=8.0 \mathrm{E}+4 \pm 1.6 \mathrm{E}+2 \mathrm{~Bq}\right)$ with a delay of one week between measurements, inhalation as route of intake, type $\mathrm{F}$ aerosols of $1 \mu \mathrm{m}$ activity median aerodynamic diameter (AMAD) for different intake scenarios (acute $v s$. chronic), different delays from start of release to measurement time and $\mathrm{SF}=1.15$ as total uncertainty associated to each thyroid monitoring data, the dose assessment resulted in 22.2 and $106.6 \mathrm{mSv}$ as lower and upper values of committed 


\section{IDOSE: Data Processing Unit for Thyroid Monitoring (RPI)}

\section{Definition of the general settings of the program. Intake shape function and age-dependent "dose per content" functions}
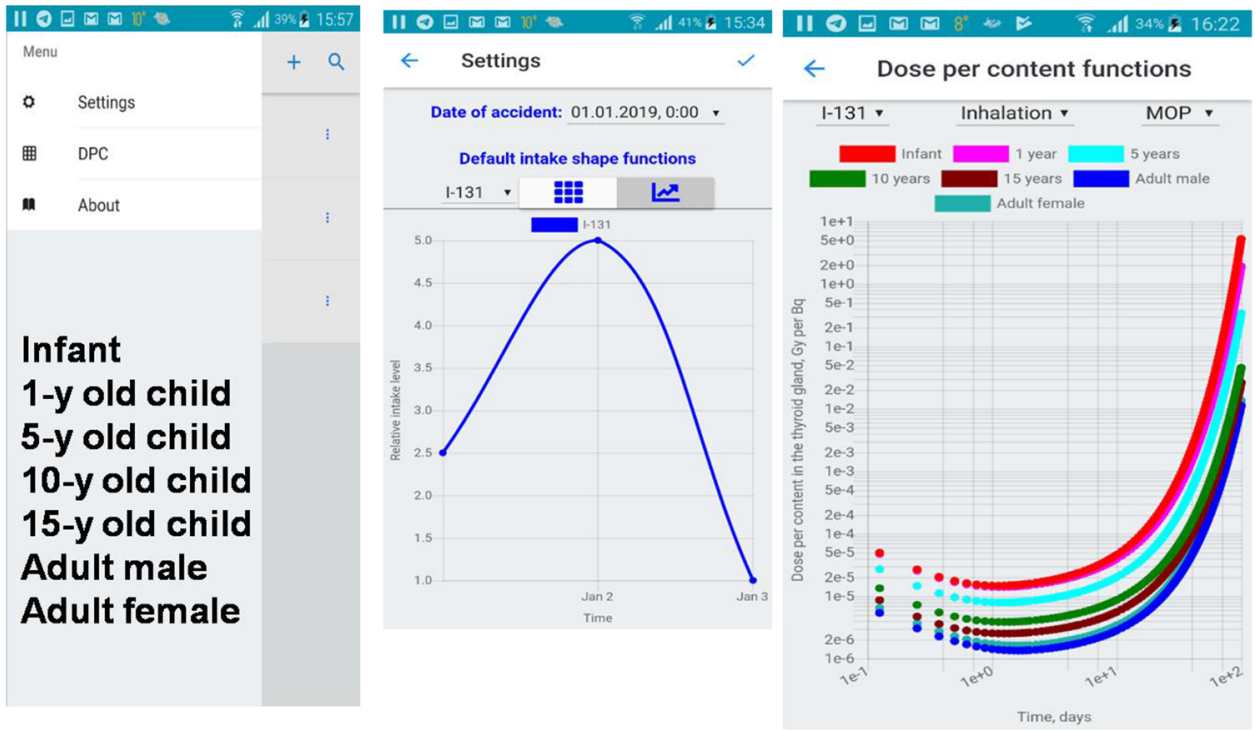

Fig. 1. IDOSE tool developed by RPI (Ukraine) for direct thyroid dose assessment from monitoring data of activity content of radioiodine in the thyroid.

equivalent dose to the thyroid respectively, considering in the latter case the correction for short-lived radionuclides other than ${ }^{131} \mathrm{I}$.

\section{Future actions}

Individual doses due to the intakes of radioiodines, obtained from thyroid monitoring may be compared with doses obtained from environmental monitoring taking into account location and exposure time of contaminated persons. On the other hand, a health risk assessment of the lifetime thyroid cancer risk from the thyroid doses may be done considering different dose estimates for adults and children, using a new tool developed in this project.

Acknowledgement. The work described in this paper was conducted within the CONFIDENCE project, which was part of the CONCERT project. This project has received funding from the Euratom research and training programme 2014-2018 under grant agreement No. 662287. The authors want to thank Pavel Fojtik (SURO, Czech Republic) for providing the data of Czech exposed person in this study, see also Lopez et al., 2016.

Disclaimer (Art. 29.5 GA). This publication reflects only the author's view. Responsibility for the information and views expressed therein lies entirely with the authors. The European Commission is not responsible for any use that may be made of the information it contains.

\section{References}

Castellani CM, Marsh JW, Hurtgen C, Blanchardon E, Berard P, Giussani A, Lopez MA. 2013. IDEAS guidelines V2 for the estimation of committed doses from incorporation monitoring data. EURADOS Report 2013-01.

Champion D, Korsakissok I, Didier D, Mathieu A, Quélo D, Groell J, Quentric E, Tombette M, Benoit J-P, Saunier O, Parache V, SimonCornu M, Gonze MA, Renaud PH, Cessac B, Navarro E, ServantPerrier A-C. 2013. The IRSN's earliest assessments of the Fukushima accident's consequences for the terrestrial environment in Japan. Radioprotection 48: 11-37.

Cauwels V, Beerten K, Vanhavere F, Lievens L. 2010. Accident dosimetry using chip cards. In: Third European IRPA Congress, Helsinki, Finland, pp. 866-874.

ICRP Publication 103. 2007. The 2007 recommendations of the International Commission on Radiological Protection. Ann. ICRP 37.

ICRP Publication 119. 2012. Compendium of dose coefficients based on ICRP publication 60. Ann. ICRP 41 (Suppl.).

ICRP Publication 130. 2015. Occupational intakes of radionuclides part 1. Ann. ICRP 44.

ICRP Publication 137. 2017. Occupational intakes of radionuclides, part 3. Ann. ICRP 46.

Kulka U, Wojcik A, Di Giorgio M, Wilkins R, Suto Y, Jang S, QuingJie L, Jiaxiang L, Ainsbury E, Woda C, Roy L, Li C, Lloyd D, Carr Z. 2019. Biodosimetry and biodosimetry networks for managing radiation emergency. Radiat. Prot. Dosim. 182: 128-138.

Kulka U, Abend M, Ainsbury E, Badie C, Francesc Barquinero J, Barrios L, Beinke C, Bortolin E, Cucu A, De Amicis A, Domínguez I, Fattibene P, Frøvig AM, Gregoire E, Guogyte K, Hadjidekova V, Jaworska A, Kriehuber R, Lindholm C, Lloyd D, Lumniczky K, Lyng F, Meschini R, Mörtl S, Monaca SD, Monteiro Gil O, Montoro A, Moquet J, Moreno M, Oestreicher U, Palitti F, Pantelias G, Patrono C, Piqueret-Stephan L, Port M, Prieto MJ, Quintens R, Ricoul M, Romm H, Roy L, Sáfrány G, Sabatier L, Sebastià N, Sommer S, Terzoudi G, Testa A, Thierens 
H, Turai I, Trompier F, Valente M, Vaz P, Voisin P, Vral A, Woda C, Zafiropoulos D, Wojcik A. 2017. RENEB - Running the European network of biological dosimetry and physical retrospective dosimetry. Int. J. Radiat. Biol. 93: 2-14.

Lopez MA, Fojtik P, Franck D, Osko J, Gerstmann U, Scholl C, Lebacq AL, Breustedt B, del Risco Norrid L. 2016. Lessons learned from the EURADOS survey on individual monitoring data and internal dose assessments of foreigners exposed in Japan following the Fukushima Daiichi NPP accident. Radiat. Prot. Dosim. 170: 402-406.

Oestreicher U, Samaga D, Ainsbury E, Antunes AC, Baeyens A, Barrios L, Beinke C, Beukes P, Blakely WF, Cucu A, De Amicis A, Depuydt J, De Sanctis S, Di Giorgio M, Dobos K, Dominguez I, Duy PN, Espinoza ME, Flegal FN, Figel M, Garcia O, Monteiro Gil O, Gregoire E, Guerrero-Carbajal C, Güçlü İ, Hadjidekova V, Hande P, Kulka U, Lemon J, Lindholm C, Lista F, Lumniczky K,
Martinez-Lopez W, Maznyk N, Meschini R, M'kacher R, Montoro A, Moquet J, Moreno M, Noditi M, Pajic J, Radl A, Ricoul M, Romm H, Roy L, Sabatier L, Sebastià N, Slabbert J, Sommer S, Stuck Oliveira M, Subramanian U, Suto Y, Que T, Testa A, Terzoudi G, Vral A, Wilkins R, Yanti L, Zafiropoulos D, Wojcik A. 2017. RENEB intercomparisons applying the conventional dicentric chromosome assay (DCA). Int. J. Radiat. Biol. 93: 20-29.

Werner CJ. (Ed.). 2017. MCNP users manual-Code version 6.2. LAUR-17-29981.

Woda C, Spöttl T. 2009. On the use of OSL of wire-bond chip card modules for retrospective and accident dosimetry. Radiat. Meas. 44: 548-553.

Wojcik A, Oestreicher U, Barrios L, Vral A, Terzoudi G, Ainsbury E, Rothkamm K, Trompier F, Kulka U. 2017. The RENEB operational basis: complement of established biodosimetric assays. Int. J. Radiat. Biol. 93: 15-19.

Cite this article as: López MA, Berkovskyy V, Ratia G, Challeton-de-Vathaire C, Davesne E, Eakins J, Franck D, Giussani A, Gregoratto D, Hernandez C, Kulka U, Mafodda A, Marsh JW, Navarro JF, Oestreicher U, Pérez B, Sierra I, Woda C. 2020. Improvements in individual dose measurement techniques following nuclear emergencies. Radioprotection 55(HS1): S89-S93 